\title{
Shifts in microbial transcription patterns prior to onset of Necrotizing enterocolitis may indicate low oxygen levels in the gut
}

\author{
Yonatan Sher, Matthew R. Olm, Tali Raveh-Sadka, Christopher Brown, Ruth Sher, Brian \\ Firek, Robyn Baker, Michael Morowitz, Jillian F. Banfield
}

\section{Highlights}

- Transcription of high oxygen affinity microbial cytochrome oxidase may predict necrotizing enterocolitis (NEC) development.

- Lower transcription of microbial genes to detoxify nitric oxide (NO) may also predict NEC development.

- Higher transcription of $\mathrm{H}_{2}$ production genes by Escherichia sp. was found in the gut of premature infants that develop NEC.

\section{Summary}

Premature infants are at risk for developing necrotizing enterocolitis (NEC), an inflammatory disease that can progress to necrosis of gut tissue. Previous attempts have failed to identify any consistent predictor of NEC. We hypothesized that prior to the appearance of NEC symptoms, the gut microbiome shifts in its transcriptional profile. To test this hypothesis we integrated genome-resolved metagenomic and metatranscriptomic data from multiple time-points in the first month of life of four preterm infants, two of whom later developed NEC. Gut microbiomes of NEC infants showed increased transcription of high oxygen affinity cytochrome oxidases and lower transcription of genes to detoxify nitric oxide, an antimicrobial compound released by host cells. These results, and high transcription of $\mathrm{H}_{2}$ production genes, suggest low $\mathrm{O}_{2}$ conditions prior to NEC onset, and are consistent with hypoxic conditions in diseased gut tissue. The findings motivate further testing of transcript data as a predictor of NEC. 
46 Necrotizing enterocolitis (NEC) is a potentially lethal gut disease that almost exclusively

47 strikes premature infants. The hallmark of NEC is inflammation of the small and/or large

48 bowel that can progress rapidly to intestinal necrosis, sepsis, and death (Hackam and

49 Caplan, 2018; Neu and Walker, 2011). Because the onset of the disease is often

50 fulminant, treatment options for severe cases are limited and often futile. Consequently,

51 in order to develop new ways to diagnose and treat NEC as early as possible, much of the

52 recent research on NEC focuses on its early development stages (Niño et al., 2016).

53 The need for disease biomarkers to enable early and accurate diagnosis motivates the

54 ongoing search for the cause of NEC. Aberrant gut bacterial colonization (Kliegman,

55 1985), or microbial dysbiosis (Warner et al., 2016), has been considered to be an

56 important risk factor for NEC. Evidence supporting the role of bacteria in NEC includes

57 positive responses to antibiotic administration, the formation of $\mathrm{H}_{2}$ (Engel et al., 1973;

58 Silverman et al., 2017), and experiments showing that NEC does not develop in germ-

59 free mice (Morowitz et al., 2010; Musemeche et al., 1986). However, while culture-

60 dependent and culture-independent studies have identified differences in fecal microbes

61 between infants with and without NEC, the observed differences have generally varied

62 across studies. Thus, it has been considered unlikely that the presence of a single

63 pathogen or absence of a single commensal is responsible for the disease. A recent meta-

64 analysis of 14 DNA-based studies reported that fecal samples from preterm infants later

65 diagnosed with NEC contained modest but statistically significant increased abundance

66 of facultative anaerobes from the Proteobacteria phylum and a modest decrease in

67 abundance of strict anaerobes (Pammi et al., 2017). As others have previously noted, an

68 understudied approach is to pair taxonomic profiling with functional information about

69 bacterial behavior in response to local conditions within the infant gut (Brown et al.,

70 2018; Pammi et al., 2017).

71 A longstanding idea has been that inadequate oxygen tension in the intestine, due to

72 reduced regional blood flow (ischemia), may be a key contributor to NEC development

73 (Morowitz et al., 2010; Young et al., 2011). In vivo patterns of microbial gene expression

74 in the infant gut may be an indicator of insufficient oxygen supply to the intestine and the

75 progression of NEC. To explore this hypothesis, we retrieved metagenomic and microbial

76 transcriptomic sequence data from prospectively collected time series of samples from

77 four premature infants, two of whom developed NEC (Fig. 1A and Fig. S1A; selected

78 from a larger metagenomic dataset, see (Brown et al., 2018)). The two NEC infants we

79 examined had microbial community compositions with a high abundance of

80 Proteobacteria phyla relative to Firmicutes phyla (Fig. S1C). To probe the response of the

81 gut microbiome to oxygen levels and other aspects of the surrounding conditions, we

82 measured transcript abundances for specific informative set of genes, and compared them

83 to transcript abundances of genes expected to have the opposite response to these

84 conditions (Fig. S1B). Transcript abundance ratios were calculated for each genome at

85 each time point, eliminating biases related to genome relative abundances (See methods

86 in supplemental). This method was chosen given the hypothesis-driven approach taken

87 here, and is distinct from more usual methods for meta-transcriptomic observations. 
88 Cytochrome oxidases are complexes of the electron transport chain that pass electrons to

$89 \mathrm{O}_{2}$, during aerobic respiration. We examined the expression of bacterial cytochrome

90 oxidase genes with different affinities for oxygen: cytochrome bd oxidase $(c y d A B)$ with

91

92

93

94

high affinity for oxygen and cytochrome o oxidase ( $c y o A B C D)$ with low affinity for oxygen (D'Mello et al., 1995, 1996). Consistent with these biochemical predictions, a previous study showed that under microaerophilic conditions, there is higher expression of $c y d A B$ whereas under aerobic conditions expression of $c y o A B C D$ genes increases (Tseng et al., 1996). In the current study we found statistically higher ratios of $c y d A B$ relative to $c y o A B C D$ transcripts in gut microbiomes of infants later diagnosed with NEC compared to the infants that did not develop NEC (Fig. 1B). This observation holds when transcript ratios are averaged for all microorganisms with these genes in each sample, and is significant when comparing across all time points $(\mathrm{p}=0.00134)$. Organisms of the genus Escherichia were examined separately, as these organisms were present in all samples and most time points. These organisms also showed higher $c y d A B$ to $c y o A B C D$ transcript ratio in the gut of NEC infants compared to control infants (Fig. 1C), and these differences were significant across all time points $(p=0.00006)$. There are two

105

106 interpretations for this result. First, bacteria in the guts of the infants that developed NEC may have had lower oxygen availability compared to those that did not develop NEC. Alternatively, the $c y d A B$ may be more highly expressed than the $c y o A B C D$ oxidase due to its function in protection against oxidative stress (Giuffrè et al., 2014). For example, the $c y o A B C D$ expression may have not changed substantially but $c y d A B$ expression was up-regulated in response to production of radical oxygen species in mitochondria (Poyton et al., 2009), which is produced in response to hypoxia.

111 To discern between these two explanations for the cytochrome oxidases expression patterns, we examined overall transcription levels of catalase-peroxidase ( $k a t G)$, a bacterial gene that encodes an enzyme known to detoxify radical oxygen species (Storz et al., 1990). Importantly, we found no significant differences in $k a t G$ expression between NEC and control samples (Fig. S2A). Furthermore, on an organism-by-organism basis, we examined the transcription levels for $c y d A B$ and $c y o A B C D$ relative to known housekeeping genes (Rocha et al., 2015) in both NEC and control infants. Expression of $c y d A B$ was comparable in both cases, whereas $c y o A B C D$ significantly varied between NEC and control infants (Fig. S2B), indicating that changes in the transcript ratio of these genes is more likely linked to low $\mathrm{O}_{2}$ availability rather than an increase in oxidative stress.

Another potential indicator of conditions in the infant gut is the availability of nitric oxide (NO). Historically, NO has been related to NEC because NO is produced as an

124 antimicrobial during inflammatory response by gut epithelial cells and as it plays a role in controlling blood flow in mesenteric vasculature (Ford et al., 1997; Nowicki et al., 2007). The microbial genetic system we used for examining microbial transcriptional response to NO is the norVW genes, coding for NO detoxifying enzymes, and their oppositely transcribed regulating gene norR (Jarboe et al., 2010). Nitric oxide binds to constitutively expressed NorR and up-regulates the transcription of nor $V W$ and down-regulates norR expression (Jarboe et al., 2010). Thus, to assess microbial transcriptional response to NO prior to NEC diagnosis, we measured the ratio of transcript abundances for nor $V W$ and norR. Higher relative transcriptional levels of NO detoxifying enzymes compared to norR were found in the guts of the control infants across all microbial community members 
133 with NO detoxification machinery (Fig. 2A), as well as for the near ubiquitous

134 Escherichia sp. (Fig. 2B).

135 Interestingly, the ratio of nor $V W$ to norR transcript abundances was higher at earlier

136 compared to later time points, for both NEC and control infants (Welch's t test,

$137 \mathrm{p}=0.0089$ ). This probably reflects the response of the preterm infant gut to burgeoning

138 microbial colonizers.

139 The ability to produce $\mathrm{H}_{2}$ is another microbial metabolism that could be an indicator of

140 oxygen levels in the premature infant gut. $\mathrm{H}_{2}$ gas, presumably of microbial origin, was

141 previously identified in mural cysts of NEC afflicted infants (Engel et al., 1973). This

142 observation led to development of a method for early detection of NEC based on breath

143 hydrogen excretion test. However, this test did not become widely accepted due to its low

144 positive prediction value (Cheu et al., 1989). A key source of microbial $\mathrm{H}_{2}$ production is

145 formate dehydrogenase $(\mathrm{FDH})$, which converts excess formate formed during mixed acid

146 fermentation into $\mathrm{CO}_{2}$ and $\mathrm{H}_{2}$ (Sawers, 2005). There are three different kinds of formate

147 dehydrogenase's that are expressed under different conditions, but only one type

148 produces $\mathrm{H}_{2}$ (Sawers, 2005). Two of the three formate dehydrogenases were found in

149 most genomes. Thus, we compared transcription of FDH genes, which are responsible for

150 formate removal from the cell and $\mathrm{H}_{2}$ production, to the transcript levels of FDO genes

151 that convert formate to $\mathrm{H}^{+}$and $\mathrm{CO}_{2}$ without production of $\mathrm{H}_{2}$. The average $\mathrm{FDH} / \mathrm{FDO}$

152 transcript ratio of the whole microbial community was not significantly different between

153 NEC and control infants (Fig. 2C). We also compared transcript ratios for members of the

154 genus Escherichia and found higher FDH compared to FDO expression in NEC

155 diagnosed infants compered to control infants (Fig. 2D). Interestingly, significantly

156 higher expression occurred at earlier time points in NEC compared to control cases.

157 Given the findings related to $\mathrm{H}_{2}$ production, we investigated genome-resolved

158 metagenomic data from five infants that developed NEC and seven that did not develop

159 NEC for whom transcriptomic data were not available. We identified genomes encoding

160 FDH and auxiliary genes and found that bacteria with the genetic capability to produce

$161 \mathrm{H}_{2}$ occurred at higher relative abundances in gut microbiomes of infants that developed

162 NEC. These bacteria were significantly more abundant in NEC infant compared to

163 control infant gut microbiomes in samples taken at 20-40 DOL (Day of life), in both the

164 smaller (Fig. S2C) and the larger meta-genomic data sets (Fig. S2B). Although

165 significant, the results are very variable. Interestingly, transcript abundance data indicate

166 capacity for higher $\mathrm{H}_{2}$ production at earlier time points (DOL 10-20) in the infants that

167 developed NEC (Fig. 2D). This finding underlines the importance of distinguishing

168 genetic capacity from activity.

169 In combination, information about $\mathrm{O}_{2}$ availability and $\mathrm{H}_{2}$ production by microbial

170 communities suggest the onset of hypoxic conditions prior to NEC formation in the gut of

171 premature infants. Pig models have shown that fetuses and premature neonates have

172 higher blood flow to the gut than in more developed gut systems (Nankervis et al., 2001),

173 and this may lead to more oxygenated conditions in the gut lumen. This is consistent with

174 previous observations showing that the gut of premature infants is initially colonized with

175 more aerobic organisms that later shifts to become more anaerobic communities (Brooks 
176 et al., 2015; La Rosa et al., 2014; Sharon et al., 2013). During NEC, however, hypoxic

177 conditions have been observed in the gut tissue of many patients (Chen et al., 2016) and

178 histologic examination of removed dead tissue demonstrates coagulation necrosis,

179 evidence for ischemic injury (Ballance et al., 1990). Yet, hypoxia is highly disputed as a

180 primary controlling factor of NEC (Chen et al., 2016; Crissinger, 1994; Neu, 2005;

181 Nowicki and Nankervis, 1994; Young et al., 2011). Recent theories on NEC development

182 point to the role of gut tissue immaturity, exhibiting high expression of Toll-like receptor

1834 (TLR4), an innate immune receptor that recognizes lipopolysaccharides on the surfaces

184 of Gram-negative bacteria. Activation of TLR4 initiates an inflammatory response, which

185 can impair intestinal microcirculation and oxygen delivery (Yazji et al., 2013). These

186 phenomena may explain the observed transcriptional response to lower $\mathrm{O}_{2}$ levels in the

187 gut microbiomes of NEC infants (Fig. 1BC). This circumstance is distinct from that that

188 occur in the mature gut, where low $\mathrm{O}_{2}$ is linked to a healthy condition and aerobicity is

189 linked to inflammation (Litvak et al., 2017).

190 Increased expression of inducible nitric oxide synthase (iNOS) in gut epithelial cells

191 often occurs as a part of the inflammatory response, and recent studies have suggested

192 that TLR4-mediated iNOS expression is a key element of NEC progression (Jilling et al.,

193 2006). Due to the short half-life of NO and the close proximity of epithelial cells to the

194 gut lumen, this is probably the main source of NO in the gut lumen (Singer et al., 1996).

195 Higher expression of iNOS in host epithelial cells was found during NEC, both in mice

196 and in gut tissue removed from NEC patients by surgery (Ford et al., 1997; Jilling et al.,

197 2006). Counter-intuitively, in this study we found down regulation of bacterial genes for

198 NO detoxification in the gut of NEC infants (Fig. 2AB), indicating lower NO levels in

199 the gut lumen.

200 Resolving these seemingly conflicting observations may require a more careful

201 examination of the factors that regulate host iNOS activity. The reaction that is catalyzed

202 by nitric oxide synthases is the conversion of L-arginine and oxygen into L-citrulline and

203 NO (Bogdan, 2015). Thus, Oxygen levels are a controlling factor for iNOS activity

204 (Bogdan, 2015). We suggest that in situations where $\mathrm{O}_{2}$ supply is reduced, the iNOS

205 response in gut epithelial cells might be frustrated and the antimicrobial response is

206 largely inactivated. Previously, a review paper also proposed that although hypoxic

207 conditions and inflammation will induce expression of iNOS gene, hypoxia would reduce

208 NO production by the iNOS enzyme (Robinson et al., 2011). However, we cannot rule

209 out the possibility that lower availability of another iNOS substrate, L-arginine, may limit

210 the activity of this enzyme during NEC development, as low L-arginine concentrations

211 are associated with NEC (Watkins and Besner, 2013).

212 In conclusion, this study shows that meta-transcriptomic data can probe physiological

213 conditions that gut microbial communities experience. Despite the small sample size, we

214 demonstrated that the transcript abundance of $\mathrm{H}_{2}$ forming formate dehydrogenase,

215 relative to non- $\mathrm{H}_{2}$ forming formate dehydrogenase, is significantly higher at early time

216 points for Escherichia sp. genomes in infants that develop NEC. The higher

217 transcriptional response to NO by gut microbiome in control compared to NEC infants

218 was even more significant. We suggest that lower NO concentrations in infants that

219 develop NEC are probably related to lower $\mathrm{O}_{2}$ availability, due to reduced blood flow 
220 that restricts NO synthesis in epithelial gut cells. Lower $\mathrm{O}_{2}$ availability also increased 221 microbial transcript ratios for the cytochrome oxidase that evolved to function in low $\mathrm{O}_{2}$ 222 environments relative to the cytochrome oxidase optimized for high $\mathrm{O}_{2}$ conditions in

223 NEC infants compared to control infants. A larger study is needed to verify these results 224 and confirm our main conclusion, that the gut microbiome, in early stages of NEC

225 development, senses and responds to different physiological conditions compared to

226 microbiomes in guts where NEC is not developing. Understanding the physiological

227 conditions during early stages of NEC development may inspire the development of new 228 tools for early NEC diagnosis, enabling treatment prior to the fast deterioration phase of 229 the disease. 


\section{ACKNOWLEDGMENTS}

This research was supported by the National Institutes of Health (NIH) under award no. RAI092531A. This work was supported in part by the March of Dimes Foundation research Grant no. 5-FY10-103 (to MJM). YS work was supported by postdoctoral fellowship grant no. 2016-67012-24717 from the USDA National Institute of Food and Agriculture. 


\section{REFFERNCES}

Ballance, W.A., Dahms, B.B., Shenker, N., and Kliegman, R.M. (1990). Pathology of neonatal necrotizing enterocolitis: a ten-year experience. J. Pediatr. 117, 6-13.

Bogdan, C. (2015). Nitric oxide synthase in innate and adaptive immunity: an update. 36, 34-45.

Brooks, B., Mueller, R.S., Young, J.C., Morowitz, M.J., Hettich, R.L., and Banfield, J.F. (2015). Strain-resolved microbial community proteomics reveals simultaneous aerobic and anaerobic function during gastrointestinal tract colonization of a preterm infant. Front. Microbiol. 6, 1-10.

Brown, C.T., Xiong, W., Olm, M.R., Thomas, B.C., Baker, R., Firek, B., Morowitz, M.J., Hettich, R.L., and Banfield, J.F. (2018). Hospitalized premature infants are colonized by related bacterial strains with distinct proteomic profiles. MBio 9.

Chen, Y., Chang, K.T.E., Lian, D.W.Q., Lu, H., Roy, S., Laksmi, N.K., Low, Y., Krishnaswamy, G., Pierro, A., and Ong, C.C.P. (2016). The role of ischemia in necrotizing enterocolitis. J. Pediatr. Surg. 51, 1255-1261.

Cheu, H.W., Brown, D.R., and Rowe, M.I. (1989). Breath Hydrogen Excretion as a Screening Test for the Early Diagnosis of Necrotizing Enterocolitis. Am. J. Dis. Child. $143,156-159$.

Crissinger, K. (1994). Regulation of hemodynamics and oxygenation in developing intestine: insight into the pathogenesis of necrotizing enterocolitis. Acta Pædiatrica 83, 810 .

D’Mello, R., Hill, S., and Poole, R.K. (1995). The oxygen affinity of cytochrome bo' in Escherichia coli determined by the deoxygenation of oxyleghemoglobin and oxymyoglobin: $\mathrm{K}(\mathrm{m})$ values for oxygen are in the submicromolar range. J. Bacteriol. 177, 867-870.

D’Mello, R., Hill, S., and Poole, R.K. (1996). The Cytochrome bd Quinol Oxidase in Escherichia coli Has an Extremely High Oxygen Affinity and Two-Oxygen-binding Haems: Implicaitons for Regulation of Activity in vivo by Oxygen Inihibition. Microbiology 142, 755-763.

Diamond, S., Andeer, P., Li, Z., Crits-Christoph, A., Burstein, D., Anantharaman, K., Lane, K.R., Thomas, B.C., Pan, C., Northen, T., et al. (2018). Processing of grassland soil $\mathrm{C}-\mathrm{N}$ compounds into soluble and volatile molecules is depth stratified and mediated by genomically novel bacteria and archaea. BioRxiv.

Engel, R.R., Virnig, N.L., Hunt, C.E., and Levitt, M.D. (1973). Origin of Mural Gas in Nectorizing Entercolitis. Pediatr. Res. 7, 64.

Ford, H.R., Watkins, S., Reblock, K., and Rowe, M. (1997). The role of inflammatory cytokines and nitric oxide in the pathogenesis of necrotizing enterocolitis. J. Pediatr. Surg. 32, 275-282.

Giuffrè, A., Borisov, V.B., Arese, M., Sarti, P., and Forte, E. (2014). Cytochrome bd oxidase and bacterial tolerance to oxidative and nitrosative stress. Biochim. Biophys. 
Acta - Bioenerg. 1837, 1178-1187.

Hackam, D., and Caplan, M. (2018). Necrotizing enterocolitis $\square$ : Pathophysiology from a historical context. Semin. Pediatr. Surg. 27, 11-18.

Hyatt, D., Chen, G.L., LoCascio, P.F., Land, M.L., Larimer, F.W., and Hauser, L.J. (2010). Prodigal: Prokaryotic gene recognition and translation initiation site identification. BMC Bioinformatics 11 .

Jarboe, L.R., Hyduke, D.R., and Liao, J.C. (2010). Chapter 4 - Systems Approaches to Unraveling Nitric Oxide Response Networks in Prokaryotes. In Nitric Oxide: Biology and Pathobiology, L.J. Ignarro, ed. (San Diego: Academic Press), pp. 103-136.

Jilling, T., Simon, D., Lu, J., Meng, F.J., Li, D., Schy, R., Thomson, R.B., Soliman, A., Arditi, M., and Caplan, M.S. (2006). The Roles of Bacteria and TLR4 in Rat and Murine Models of Necrotizing Enterocolitis. J. Immunol. 177, 3273-3282.

Kliegman, R.M. (1985). The role of Clostridia in the pathogenesis of neonatal necrotizing enterocolitis. In Clostridia in Gastrointestinal Disease, P. Borriello, ed. (Boca Raton: CRC Press.), pp. 67-87.

Litvak, Y., Byndloss, M.X., Tsolis, R.M., and Bäumler, A.J. (2017). Dysbiotic Proteobacteria expansion: a microbial signature of epithelial dysfunction. Curr. Opin. Microbiol. 39, 1-6.

Martin, M. (2011). Cutadapt removes adapter sequences from high-throughput sequencing reads. EMBnet.Journal 17, 10.

Morowitz, M.J., Poroyko, V., Caplan, M., Alverdy, J., and Liu, D.C. (2010). Redefining the Role of Intestinal Microbes in the Pathogenesis of Necrotizing Enterocolitis. Pediatrics 125, 777-785.

Musemeche, C.A., Kosloske, A.M., Bartow, S.A., and Umland, E.T. (1986). Comparative efects of ischemia, bacteria, and substrate on the pathogenesis of intestinal necrosis. J. Pediatr. Surg. 21, 536-538.

Nankervis, C.A., Reber, K.M., and Nowicki, P.T. (2001). Age-Dependent Changes in the Postnatal Intestinal Microcirculation. Microcirculation 8, 377-387.

Neu, J. (2005). The "myth" of asphyxia and hypoxia-ischemia as primary causes of necrotizing enterocolitis. Biol. Neonate 87, 97-98.

Neu, J., and Walker, A.W. (2011). Necrotizing Enterocolitis. N. Engl. J. Med. 38, 552559.

Niño, D.F., Sodhi, C.P., and Hackam, D.J. (2016). Necrotizing enterocolitis: new insights into pathogenesis and mechanisms. Nat. Rev. Gastroenterol. Hepatol. 13, 590-600.

Nowicki, P.T., and Nankervis, C.A. (1994). The Role of the Circulation in the Pathogenesis of Necrotizing Enterocolitis. Clin. Perinatol. 21, 219-234.

Nowicki, P.T., Caniano, D.A., Hammond, S., Giannone, P.J., Besner, G.E., Reber, K.M., and Nankervis, C.A. (2007). Endothelial nitric oxide synthase in human intestine resected for necrotizing enterocolitis. J. Pediatr. 150, 40-45.

Olm, M.R., Brown, C.T., Brooks, B., and Banfield, J.F. (2017). DRep: A tool for fast and 
accurate genomic comparisons that enables improved genome recovery from metagenomes through de-replication. ISME J. 11, 2864-2868.

Pammi, M., Cope, J., Tarr, P.I., Warner, B.B., Morrow, A.L., Mai, V., Gregory, K.E., Simon Kroll, J., McMurtry, V., Ferris, M.J., et al. (2017). Intestinal dysbiosis in preterm infants preceding necrotizing enterocolitis: A systematic review and meta-analysis. Microbiome 5.

Peng, Y., Leung, H.C.M., Yiu, S.M., and Chin, F.Y.L. (2012). IDBA-UD: A de novo assembler for single-cell and metagenomic sequencing data with highly uneven depth. Bioinformatics $28,1420-1428$.

Poyton, R.O., Ball, K.A., and Castello, P.R. (2009). Mitochondrial generation of free radicals and hypoxic signaling. Trends Endocrinol. Metab. 20, 332-340.

Raveh-Sadka, T., Thomas, B.C., Singh, A., Firek, B., Brooks, B., Castelle, C.J., Sharon, I., Baker, R., Good, M., Morowitz, M.J., et al. (2015). Gut bacteria are rarely shared by co-hospitalized premature infants, regardless of necrotizing enterocolitis development. Elife 2015, 1-25.

Robinson, M.A., Baumgardner, J.E., and Otto, C.M. (2011). Oxygen-dependent regulation of nitric oxide production by inducible nitric oxide synthase. Free Radic. Biol. Med. 51, 1952-1965.

Rocha, D.J.P., Santos, C.S., and Pacheco, L.G.C. (2015). Bacterial reference genes for gene expression studies by RT-qPCR: survey and analysis. Antonie Van Leeuwenhoek $108,685-693$.

La Rosa, P.S., Warner, B.B., Zhou, Y., Weinstock, G.M., Sodergren, E., Hall-moore, C.M., Stevens, H.J., Bennett, W.E., Shaikh, N., Linneman, L.A., et al. (2014). Patterned progression of bacterial populations in the premature infant gut. Proc. Natl. Acad. Sci. 111, 17336-17336.

Sawers, R.G. (2005). Formate and its role in hydrogen production in Escherichia coli. Biochem Soc Trans 33, 42-46.

Sharon, I., Morowitz, M.J., Thomas, B.C., Costello, E.K., Relman, D.A., and Banfield, J.F. (2013). Time series community genomics analysis reveals rapid shifts in bacterial species, strains, and phage during infant gut colonization. Genome Res. 23, 111-120.

Sieber, C.M.K., Probst, A.J., Sharrar, A., Thomas, B.C., Hess, M., Tringe, S.G., and Banfield, J.F. (2018). Recovery of genomes from metagenomes via a dereplication, aggregation and scoring strategy. Nat. Microbiol. 3, 836-843.

Silverman, M.A., Konnikova, L., and Gerber, J.S. (2017). Impact of Antibiotics on Necrotizing Enterocolitis and Antibiotic-Associated Diarrhea. Gastroenterol. Clin. North Am. 46, 61-76.

Singer, I., Kawka, D.W., Scott, S., Weidner, J.R., Mumford, R. a, Riehl, T.E., Stenson, W.F., Singer, I.I., Kawka, D.W., Scott, S., et al. (1996). Expression of inducible nitric oxide synthase and nitrotyrosine in colonic epithelium in inflammatory bowel disease. Gastroenterology 111, 871-885.

Storz, G., Tartaglia, L.A., Farr, S.B., and Ames, B.N. (1990). Bacterial defenses against 
oxidative stress. Trends Genet. 6, 363-368.

Tseng, C.P., Albrecht, J., and Gunsalus, R.P. (1996). Effect of microaerophilic cell growth conditions on expression of the aerobic (cyoABCDE and cydAB) and anaerobic (narGHJI, frd $\mathrm{ABCD}$, and dms $\mathrm{ABC}$ ) respiratory pathway genes in Escherichia coli. J Bacteriol 178, 1094-1098.

Warner, B.B., Deych, E., Zhou, Y., Hall-Moore, C., Weinstock, G.M., Sodergren, E., Shaikh, N., Hoffmann, J.A., Linneman, L.A., Hamvas, A., et al. (2016). Gut bacteria dysbiosis and necrotising enterocolitis in very low birthweight infants: A prospective case-control study. Lancet 387, 1928-1936.

Watkins, D.J., and Besner, G.E. (2013). The role of growth factors in intestinal regeneration and repair in necrotizing enterocolitis. Semin. Pediatr. Surg. 22, 83-87.

Yazji, I., Sodhi, C.P., Lee, E.K., Good, M., Egan, C.E., Afrazi, A., Neal, M.D., Jia, H., Lin, J., Ma, C., et al. (2013). Endothelial TLR4 activation impairs intestinal microcirculatory perfusion in necrotizing enterocolitis via eNOS-NO-nitrite signaling. Proc. Natl. Acad. Sci. 110, 9451-9456.

Young, C.M., Kingma, S.D.K., and Neu, J. (2011). Ischemia-reperfusion and neonatal intestinal injury. J. Pediatr. 158, e25-e28. 


\section{FIGURE CAPTIONS}

Figure 1: Sampling scheme and microbial transcriptional response to oxygen by gut microbiome of NEC and control premature infants.

A) Feces sampling scheme: check mark indicate which infant and at what day of life feces were taken. Red lines on the NEC infant's rows indicate the day of life (DOL) NEC was detected. DOL10-19 were collapsed into the $1^{\text {st }}$ time block and DOL 20-37 into the $2^{\text {nd }}$ time block.

B) Box plots showing the ratios of $c y d A B$ and $c y o A B C D$ transcript abundances averaged over all genomes possessing both sets of genes. Transcript ratios were compared between NEC and control infants in each time block (short lines above) and across all time points (longer lines). Distributions of transcript ratios were compared using Welch's t-test with Bonferroni correction. Asterisks and double asterisks $(*, * *)$ represent $p<0.05$ and $p<0.01$, respectively.

C) Box plots showing the ratios of $c y d A B$ and $c y o A B C D$ transcript abundances for genomes of bacteria of the genus Escherichia. For statistical methods, see (B).

Figure 2: Microbial transcriptional responses to nitric oxide and $\mathrm{H}_{2}$ production by gut microbiomes of NEC and control premature infants.

A) Box plots showing the ratios of norVW and norR transcript abundances averaged over all genomes possessing both set of genes. Distributions of transcript ratios were compared using non-parametric Wilcoxon rank-sum test with Bonferroni correction. Asterisks and double asterisks (*,**) represent $p<0.05$ and $p<0.01$, respectively.

B) Box plots showing the ratios of norVW and norR transcript abundances for genomes of the genus Escherichia. For statistical methods, see Figure 1B caption.

C) Box plots showing transcript ratios between $F D H$ and $F D O$ averaged between all genomes possessing both set of genes. For statistical methods, see Figure 1B caption.

D) Box plots showing the ratios of $F D H$ and $F D O$ transcript abundances in genomes of the genus Escherichia. Distributions of transcript ratio were compared using Welch's t-test (on $\log 10$ transformed data) with Bonferroni correction. Asterisks and double asterisks $(*, * *)$ represent $p<0.05$ and $p<0.01$, respectively. 


\begin{tabular}{|c|c|c|c|c|c|c|c|c|c|c|c|c|c|c|c|c|c|c|c|}
\hline A) & Baby & & & & $1^{\text {st }} \mathrm{Tin}$ & Bloc & & & & & & & & ${ }^{n d} \mathrm{Tir}$ & Bloc & & & & \\
\hline \multirow{2}{*}{ NEC } & & $\checkmark$ & & & & $\checkmark$ & & & $\checkmark$ & & $\checkmark$ & $\checkmark$ & & & & & & & \\
\hline & 69 & & & & $\checkmark$ & & & $\checkmark$ & & & & & $\checkmark$ & $\checkmark$ & & & & & \\
\hline \multirow{3}{*}{ Control } & 66 & & & & & & & $\checkmark$ & $\checkmark$ & & & & & & $\checkmark$ & & $\checkmark$ & & $\checkmark$ \\
\hline & 64 & & $\checkmark$ & $\checkmark$ & & & $\checkmark$ & & & $\checkmark$ & & & & & & & & $\checkmark$ & \\
\hline & & 10 & 11 & 13 & 14 & 15 & 16 & 17 & 19 & 20 & 21 & 25 & 27 & 28 & 31 & 32 & 33 & 36 & 37 \\
\hline \multicolumn{20}{|c|}{ Days of life } \\
\hline
\end{tabular}
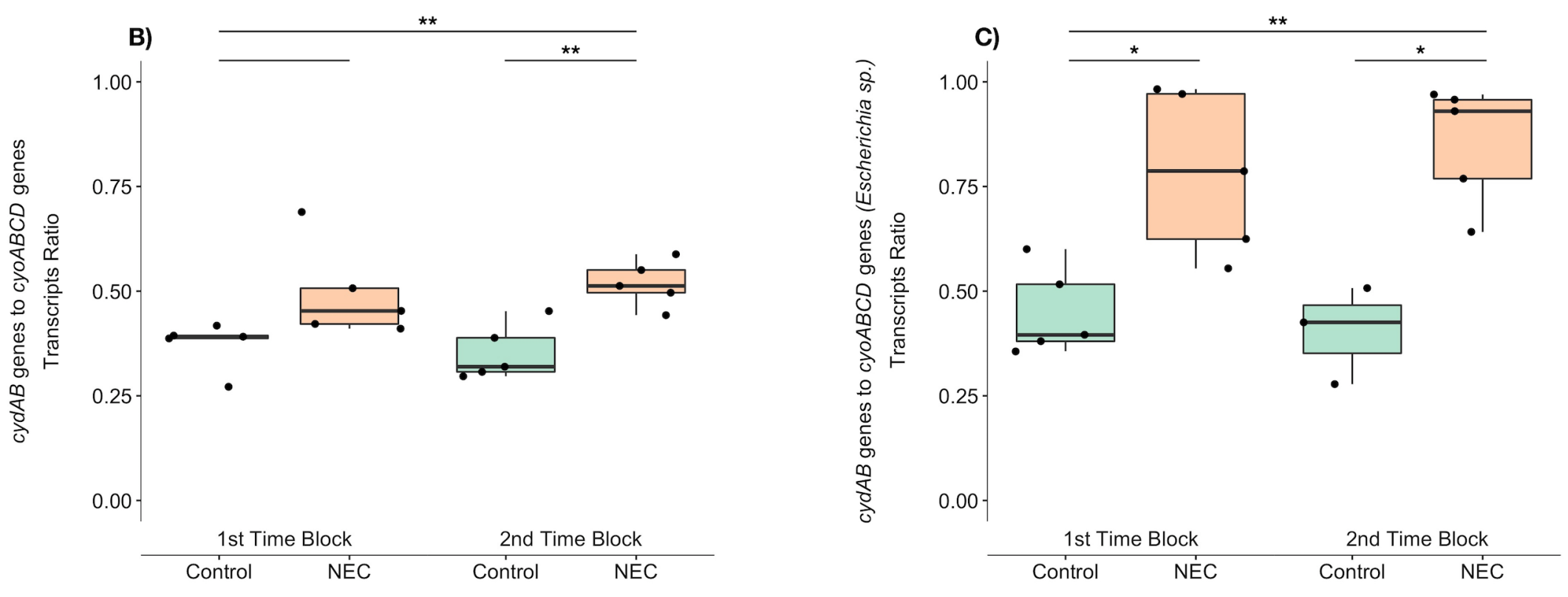

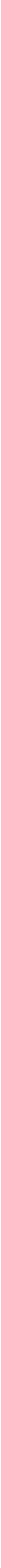